\title{
Global Asymptotic Stability of 3-Species Mutualism Models with Diffusion and Delay Effects
}

\author{
Chang-you Wang, ${ }^{1,2,3}$ Shu Wang, ${ }^{3}$ and Xiang-ping Yan ${ }^{4}$ \\ ${ }^{1}$ College of Mathematics and Physics, Chongqing University of Posts and Telecommunications, \\ Chongqing 400065, China \\ ${ }^{2}$ Key Laboratory of Network control E Intelligent Instrument, (Chongqing University of Posts and \\ Telecommunications), Ministry of Education, Chongqing 400065, China \\ ${ }^{3}$ College of Applied Sciences, Beijing University of Technology, Beijing 100124, China \\ ${ }^{4}$ Department of Mathematics, Lanzhou Jiaotong University, Lanzhou 730070, China
}

Correspondence should be addressed to Chang-you Wang, wangcy@cqupt.edu.cn

Received 21 March 2009; Accepted 6 June 2009

Recommended by Leonid Berezansky

In this paper, the Lotka-Volterra 3-species mutualism models with diffusion and delay effects is investigated. A simple and easily verifiable condition is given to ensure the global asymptotic stability of the unique positive steady-state solution of the corresponding steady-state problem in a bounded domain with Neumann boundary condition. Our approach to the problem is based on inequality skill and the method of the upper and lower solutions for a more general reactiondiffusion system. Finally, some numerical simulations are given to illustrate our results.

Copyright (C) 2009 Chang-you Wang et al. This is an open access article distributed under the Creative Commons Attribution License, which permits unrestricted use, distribution, and reproduction in any medium, provided the original work is properly cited.

\section{Introduction}

Modeling and analysis of the dynamics of biological populations by means of differential equations is one of the primary concerns in population growth problems. A well-known and extensively studied class of models in population dynamics is the Lotka-Volterra system which models the interaction among various species. In the earlier literature most of the discussions are devoted to coupled systems of two equations (cf. [1-11]). In the recent years, attention has been given to reaction-diffusion systems with three population species, and the main concerns in these works are the prey-predator and competition models with or without time delays (cf. [12-24]). The purpose of this paper is to investigate the asymptotic behavior of the time-dependent solution for a 3-species mutualism model, where the effect of diffusion and time delays is both taken into consideration by obtaining existence of positive solution for the corresponding inequalities. The system of equations under consideration is the LotkaVolterra 3-species mutualism model, where the population densities do not move across the 
boundary of a habitat, and time delays may appear in the opposing species; the densities of three populations $u, v$, and $w$ are governed by the following coupled equations:

$$
\begin{gathered}
\frac{\partial u}{\partial t}-d_{1} L_{1} u=u\left(a_{1}-c_{1} u+e_{1} v+\beta_{1} J_{2} * v\right), \\
\frac{\partial v}{\partial t}-d_{2} L_{2} v=v\left(a_{2}+b_{2} u-c_{2} v+e_{2} w+\alpha_{2} J_{1} * u+\beta_{2} J_{3} * w\right), \\
\frac{\partial w}{\partial t}-d_{3} L_{3} w=w\left(a_{3}+e_{3} v-c_{3} w+\beta_{3} J_{2} * v\right) \quad(x \in \Omega, t>0),
\end{gathered}
$$

with the boundary and initial conditions

$$
\begin{gathered}
\frac{\partial u(x, t)}{\partial n}=\frac{\partial v(x, t)}{\partial n}=\frac{\partial w(x, t)}{\partial n}=0 \quad(x \in \partial \Omega, t>0), \\
u(x, t)=\eta_{1}(x, t) \quad\left(t \in I_{1}\right), \quad v(x, t)=\eta_{2}(x, t) \quad\left(t \in I_{2}\right), \\
w(x, t)=\eta_{3}(x, t) \quad\left(t \in I_{3}, x \in \Omega\right) .
\end{gathered}
$$

Here $\Omega$ is a bounded domain in $R^{n}$ with boundary $\partial \Omega, \partial / \partial n$ denotes the outward normal derivative on $\partial \Omega$, and $\eta_{i}(x, t)$ is a smooth function. The above problem (1.1)-(1.2) arises in a simple food chain describing three interacting species in a spatial habitat $\Omega$. For each $i=$ $1,2,3, a_{i}, c_{i}$ are positive constants. $b_{i}, e_{i}, \alpha_{i}$, and $\beta_{i}$ are nonnegative constants with $e_{i}^{*} \equiv e_{i}+\beta_{i}>$ $0, b_{2}^{*} \equiv b_{2}+\alpha_{2}>0$, and its respective diffusion rate is denoted by $d_{i}$. The real number $a_{i}$ is the net birth rate of the $i$ th species, and $c_{i}$ is its respective intraspecific competition. The parameters $b_{i}, e_{i}, \alpha_{i}$ and $\beta_{i}$ are interspecific cooperation. $L_{i}$ is a uniformly elliptic operator in the form

$$
L_{i} u=-\sum_{j, k=1}^{N} a_{j, k}^{(i)}(x, t) \frac{\partial^{2} u}{\partial x_{j} \partial x_{k}}+\sum_{j=1}^{N} b_{j}^{(i)}(x, t) \frac{\partial u}{\partial x_{j}} \quad(i=1,2,3)
$$

(cf. [1]). The functions $J_{i} * u_{i}, i=1,2,3$, with $\left(u_{1}, u_{2}, u_{3}\right)=(u, v, w)$ are given either by the discrete time delay

$$
J_{i} * u_{i}=u_{i}\left(t-\tau_{i}, x\right)
$$

or by the continuous time delay

$$
J_{i} * u_{i}=\int_{-\tau_{i}}^{t} J_{i}(t-s) u_{i}(s, x) d s
$$

and the interval $I_{i}$ is given by $I_{i}=\left[-\tau_{i}, 0\right]$, where $\tau_{i}>0$ is a constant representing the time delay. It is allowed that the type of time delays and the values of $\tau_{i}$ may be different for different $u_{i}$. This consideration includes various combination of discrete and continuous time delays for the species $u, v$, and $w$. 
Throughout the paper we assumed that the function $J_{i}(t)$ is piecewise continuous in $R_{+} \equiv[0,+\infty)$ and possesses the property

$$
\begin{gathered}
J_{i}(t) \geq 0 \quad \text { for } t \geq 0, \quad J_{i}(t) \equiv 0 \quad \text { for } t>\tau_{i}, \\
\int_{0}^{\tau_{i}} J_{i}(t) d t=1 \quad(i=1,2,3) .
\end{gathered}
$$

The above property implies that for any constant function $u_{i}(t, x)=\rho_{i}$, we have

$$
\int_{-\tau_{i}}^{t} J_{i}(t-s) u_{i}(s, x) d s=\rho_{i} \quad(t \geq 0)
$$

The same is obviously true if $J_{i} * u_{i}$ is given by (1.4). It is also assumed that the domain $\Omega$ is smooth and the coefficients $a_{j, k}^{(i)} b_{j}^{(i)}$ of $L_{i}$ are smooth functions in $\Omega$ (cf. [12]). In the special case of the diffusion operator $L_{i} u_{i}=D_{i}(x) D^{2} u_{i}$, it suffices to assume that $D_{i}(x)$ is strictly positive on $\bar{\Omega}$ and in $C^{1+\alpha}(\bar{\Omega})$ for some $\alpha \in(0,1)$. We allow $L_{i}=0$ (and without the corresponding boundary condition) for some or all $i$. In particular, if $L_{i} u_{i} \equiv 0$ for all $i$ then the equations in (1.1)-(1.2) are reduced to the ordinary differential system (with time delays)

$$
\begin{gathered}
\frac{d u}{d t}=u\left(a_{1}-c_{1} u+e_{1} v+\beta_{1} J_{2} * v\right) \quad(t>0), \\
\frac{d v}{d t}=v\left(a_{2}+b_{2} u-c_{2} v+e_{2} w+\alpha_{2} J_{1} * u+\beta_{2} J_{3} * w\right) \quad(t>0), \\
\frac{d w}{d t}=w\left(a_{3}+e_{3} v-c_{3} w+\beta_{3} J_{2} * v\right) \quad(t>0), \\
u(t)=\eta_{1}(t) \quad\left(t \in I_{1}\right), \quad v(t)=\eta_{2}(t) \quad\left(t \in I_{2}\right), \\
w(x, t)=\eta_{3}(t) \quad\left(t \in I_{3}\right) .
\end{gathered}
$$

Problem (1.8) and various similar problems have been investigated by many investigators in the framework of ordinary differential systems (cf. [25-28] and references therein). It is to be noted that if $\Delta u_{i} \neq 0$ and $\alpha_{i}=\beta_{i}=0$ for every $i$ then problem (1.1) is reduced to the following 3-species mutualism models without time delays:

$$
\begin{gathered}
u_{1 t}-d_{1} \Delta u_{1}=u_{1}\left(a_{1}-c_{1} u_{1}+e_{1} u_{2}\right), \quad \text { in } \Omega_{T}, \\
u_{2 t}-d_{2} \Delta u_{2}=u_{2}\left(a_{2}+b_{2} u_{1}-c_{2} u_{2}+e_{2} u_{3}\right), \quad \text { in } \Omega_{T}, \\
u_{3 t}-d_{3} \Delta u_{3}=u_{3}\left(a_{3}+b_{3} u_{2}-c_{3} u_{3}\right), \quad \text { in } \Omega_{T}, \\
u_{1}(x, t)=u_{2}(x, t)=u_{3}(x, t)=0, \quad \text { on } \partial \Omega_{T}, \\
u_{i}(x, 0)=u_{i, 0}(x), \quad \text { for } i=1,2,3, \quad \text { in } \Omega,
\end{gathered}
$$


where the domains $\Omega_{T}$ and $\partial \Omega_{T}$ are defined as, respectively, $\Omega \times(0, T)$ and $\partial \Omega \times(0, T)$. Here $\Omega$ is a bounded domain in $R^{N}$ with smooth boundary $\partial \Omega$. T is the maximal existence time of the solution. $u_{i, 0}$ is a smooth function satisfying the compatibility condition $u_{i, 0}(x)=0$ for $x \in \partial \Omega$. The meaning of the parameters of systems (1.9) is as same as those of (1.1)-(1.2). Kim and Lin [22] proved that global solutions of (1.9) exist if the intraspecific competitions are strong; whereas blow-up solutions exist under certain conditions of that the intraspecific competitions are weak, and [23] obtained the upper bound of blow-up rate for any $N$ and the lower bound of blow-up rate for $N=1$. On the other hand, if $e_{2}=\beta_{2}=0$ then it reduces to the 2-species cooperating models, which have been extensively investigated in the current literature (cf. $[2,10,11]$ and references therein). Our conclusion about the global asymptotic stability of a positive steady-state solution to system (1.1)-(1.2) is directly applicable to the above special cases (see Corollary 3.2).

This work is motivated from the following two-prey one-predator model:

$$
\begin{gathered}
\frac{\partial u}{\partial t}-L_{1} u=a_{1}(x) u\left(1-u-b_{1} v-c_{1} w-\beta_{1} J_{2} * v-\gamma_{1} J_{3} * w\right) \\
\frac{\partial v}{\partial t}-L_{2} v=a_{2}(x) v\left(1-v-b_{2} u-c_{2} w-\beta_{2} J_{1} * u-\gamma_{2} J_{3} * w\right) \\
\frac{\partial w}{\partial t}-L_{3} w=a_{3}(x) w\left(1-w+b_{3} u+c_{3} v+\beta_{3} J_{1} * u+\gamma_{3} J_{2} * v\right) \quad(x \in \Omega, t>0), \\
\frac{\partial u}{\partial n}=\frac{\partial v(x, t)}{\partial n}=\frac{\partial w(x, t)}{\partial n}=0 \quad(x \in \partial \Omega, t>0) \\
u(x, t)=\eta_{1}(x, t) \quad\left(t \in I_{1}\right), v(x, t)=\eta_{2}(x, t) \quad\left(t \in I_{2}\right) \\
w(x, t)=\eta_{3}(x, t) \quad\left(t \in I_{3}, x \in \Omega\right)
\end{gathered}
$$

and one-prey two-predator model

$$
\begin{gathered}
\frac{\partial u}{\partial t}-L_{1} u=a_{1}(x) u\left(1-u-b_{1} v-c_{1} w-\beta_{1} J_{2} * v-\gamma_{1} J_{3} * w\right), \\
\frac{\partial v}{\partial t}-L_{2} v=a_{2}(x) v\left(1-v+b_{2} u-c_{2} w+\beta_{2} J_{1} * u-\gamma_{2} J_{3} * w\right) \\
\frac{\partial w}{\partial t}-L_{3} w=a_{3}(x) w\left(1-w+b_{3} u-c_{3} v+\beta_{3} J_{1} * u-\gamma_{3} J_{2} * v\right) \quad(x \in \Omega, t>0), \\
\frac{\partial u}{\partial n}=\frac{\partial v(x, t)}{\partial n}=\frac{\partial w(x, t)}{\partial n}=0 \quad(x \in \partial \Omega, t>0) \\
u(x, t)=\eta_{1}(x, t) \quad\left(t \in I_{1}\right), \quad v(x, t)=\eta_{2}(x, t) \quad\left(t \in I_{2}\right) \\
w(x, t)=\eta_{3}(x, t) \quad\left(t \in I_{3}, x \in \Omega\right)
\end{gathered}
$$


as well as three-species food-chain model

$$
\begin{gathered}
\frac{\partial u}{\partial t}-L_{1} u=a_{1}(x) u\left(1-u-b_{1} v-c_{1} w-\beta_{1} J_{2} * v-\gamma_{1} J_{3} * w\right), \\
\frac{\partial v}{\partial t}-L_{2} v=a_{2}(x) v\left(1-v+b_{2} u-c_{2} w+\beta_{2} J_{1} * u-\gamma_{2} J_{3} * w\right), \\
\frac{\partial w}{\partial t}-L_{3} w=a_{3}(x) w\left(1-w+b_{3} u+c_{3} v+\beta_{3} J_{1} * u+\gamma_{3} J_{2} * v\right) \quad(x \in \Omega, t>0), \\
\frac{\partial u}{\partial n}=\frac{\partial v(x, t)}{\partial n}=\frac{\partial w(x, t)}{\partial n}=0 \quad(x \in \partial \Omega, t>0), \\
u(x, t)=\eta_{1}(x, t) \quad\left(t \in I_{1}\right), \quad v(x, t)=\eta_{2}(x, t) \quad\left(t \in I_{2}\right), \\
w(x, t)=\eta_{3}(x, t) \quad\left(t \in I_{3}, x \in \Omega\right) .
\end{gathered}
$$

Pao [29] obtained some simple and easily verifiable conditions for the existence and global asymptotic stability of a positive steady-steady solution for each of the above three-model problems.

Based on the above results, we are mainly interested in studying the asymptotic behavior of the solution of (1.1)-(1.2). The corresponding steady-state problem is given by

$$
\begin{aligned}
-d_{1} L_{1} u & =u\left(a_{1}-c_{1} u+e_{1} v+\beta_{1} v\right) \quad(x \in \Omega), \\
-d_{2} L_{2} v & =v\left(a_{2}+b_{2} u-c_{2} v+e_{2} w+\alpha_{2} u+\beta_{2} w\right) \quad(x \in \Omega), \\
-d_{3} L_{3} w & =w\left(a_{3}+e_{3} v-c_{3} w+\beta_{3} v\right) \quad(x \in \Omega), \\
\frac{\partial u}{\partial n} & =\frac{\partial v}{\partial n}=\frac{\partial w}{\partial n}=0 \quad(x \in \partial \Omega) .
\end{aligned}
$$

It is clear that the above system has always the trivial solution $(0,0,0)$ and various forms of constant semitrivial solutions, that is, constant nonnegative solutions with at least one component zero and one component positive. Our aim is to prove the existence and global asymptotic stability of a positive constant steady-state solution in the mutualism model (with respect to nonnegative initial perturbations). This global asymptotic stability result implies that system (1.1)-(1.2) is permanent, the trivial and all forms of semitrivial solutions are unstable, and the nonuniform steady-state solution does not exist.

This paper is arranged as follows. In Section 2, we give some preliminary results for a more general time-delayed reaction-diffusion system. The main results and their proofs are given in Section 3. Some numerical simulations are shown in Section 4 to illustrate our theoretical analysis. 


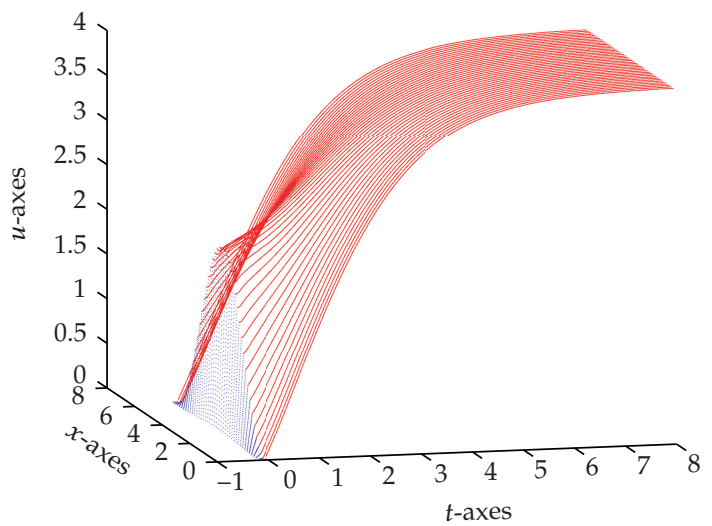

(a)

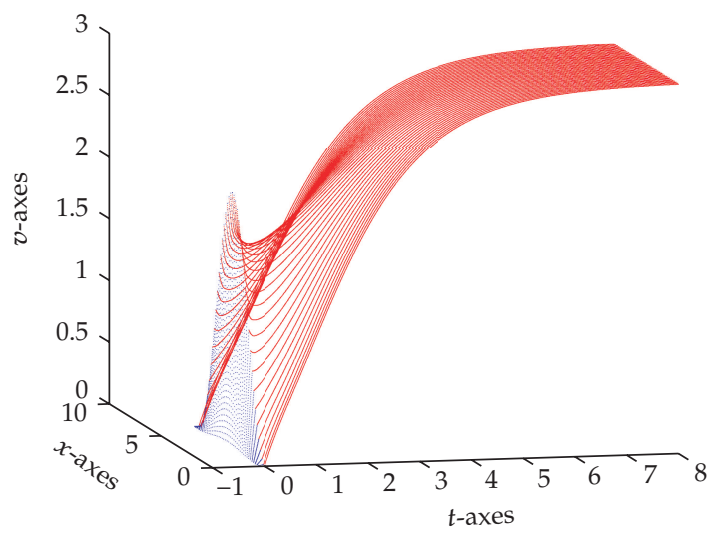

(b)

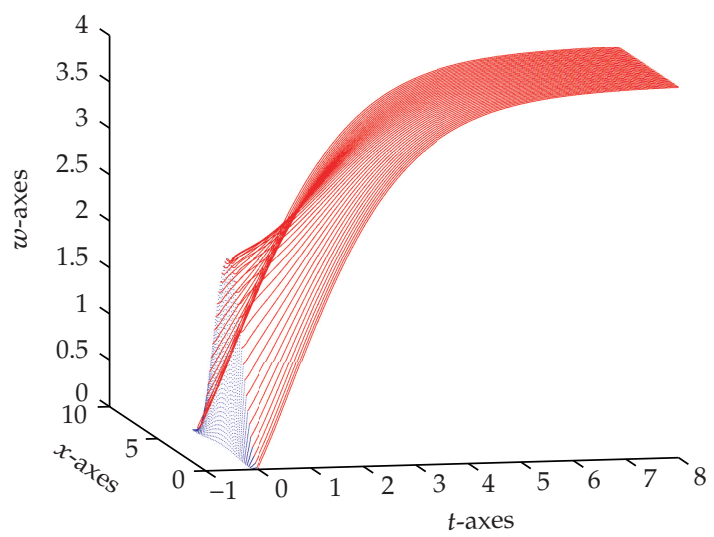

(c)

Figure 1: The solution of system (4.1). 


\section{Some Preliminary Results}

To prove the main results in this paper we first give some preliminary results for a more general time-delayed parabolic system in the form

$$
\begin{aligned}
\frac{\partial u_{i}}{\partial t}-L_{i} u_{i} & =f_{i}(x, U, J * U) \quad(x \in \Omega, t>0), \\
\frac{\partial u_{i}}{\partial n} & =0 \quad(x \in \partial \Omega, t>0), \\
u_{i}(x, t) & =\eta_{i}(x, t) \quad\left(x \in \Omega, t \in I_{i}\right), i=1,2, \ldots, N,
\end{aligned}
$$

where $U=\left(u_{1}, u_{2}, \ldots, u_{N}\right), J * U=\left(J_{1} * u_{1}, J_{2} * u_{2}, \ldots, J_{N} * u_{N}\right)$, and for each $i=$ $1,2, \ldots, N, f_{i}(\cdot, U, J * U)$ is, in general, a nonlinear function of $U$ and $J * U$, and $L_{i}$ is a uniformly elliptic operator in the form of (1.3). The components $J_{i} * u_{i}$ of $J * U$ are given by (1.4) for $i=1,2, \ldots, n_{0}$ and by (1.5) for $i=n_{0}+1, \ldots, N$, where $n_{0}$ is a nonnegative integer. (The case $n_{0}=0$ corresponds to (1.5) for every $i=1,2, \ldots, N$.)

By writing $U$ and $V$ in the split forms $U \equiv\left(u_{i},[u]_{a_{i}},[u]_{b_{i}}\right)$ and $V \equiv\left([v]_{c_{i}},[v]_{d_{i}}\right)$, respectively, where $a_{i}, b_{i}, c_{i}$, and $d_{i}$ are nonnegative integers satisfying

$$
a_{i}+b_{i}=N-1, \quad c_{i}+d_{i}=N \quad(i=1,2, \ldots, N),
$$

and $[w]_{\delta_{i}}$ denotes a vector with $\delta_{i}$ components of $w$, we write $f_{i}(x, U, V)$ in the form

$$
f_{i}(x, U, V)=f_{i}\left(x, u_{i},[u]_{a_{i}}[u]_{b_{i^{\prime}}}[v]_{c_{i^{\prime}}}[v]_{d_{i}}\right) \text { for every } i=1,2, \ldots, N
$$

Definition 2.1. We say that the vector function $F(\cdot, U, V) \equiv\left(f_{1}(\cdot, U, V), f_{2}(\cdot, U, V)\right.$, $\ldots, f_{N}(\cdot, U, V)$ ) possesses a mixed quasimonotone property in a subset $S$ of $R^{N}$, if for each $i$ there exist nonnegative integers $a_{i}, b_{i}, c_{i}$, and $d_{i}$ satisfying (2.2) such that $f_{i}\left(\cdot, u_{i},[u]_{a_{i}},[u]_{b_{i}},[v]_{c_{i}},[v]_{d_{i}}\right)$ is nondecreasing in $[u]_{a_{i},}[v]_{c_{i}}$ and is nonincreasing in $[u]_{b_{i}},[v]_{d_{i}}$ for every $U, V$ in $S$ (cf. $\left.[1,12]\right)$. In particular, if $b_{i}=d_{i}=0$ for all $i$ then $F(\cdot, U, V)$ is said to be quasimonotone nondecreasing in $S$. It is easily seen that the reaction functions in the models (1.1) are quasimonotone nondecreasing in $R_{+}^{N}$.

Definition 2.2. For quasimonotone nondecreasing functions $F(\cdot, U, V)$ we call a pair of smooth functions $\tilde{U} \equiv\left(\tilde{u}_{1}, \widetilde{u}_{2}, \ldots, \tilde{u}_{N}\right), \widehat{U} \equiv\left(\widehat{u}_{1}, \widehat{u}_{2}, \ldots, \widehat{u}_{N}\right)$ coupled upper and lower solutions of (2.1) if $\tilde{U}>\widehat{U}$ and if

$$
\begin{gathered}
\frac{\partial \tilde{u}_{i}}{\partial t}-L_{i} \tilde{u}_{i} \geq f_{i}(x, \tilde{U}, J * \tilde{U}) \quad(x \in \Omega, t>0), \\
\frac{\partial \widehat{u}_{i}}{\partial t}-L_{i} \widehat{u}_{i} \leq f_{i}(x, \widehat{U}, J * \widehat{U}) \quad(x \in \Omega, t>0), \\
\frac{\partial \tilde{u}_{i}}{\partial n} \geq 0 \geq \frac{\partial \widehat{u}_{i}}{\partial n} \quad(x \in \partial \Omega, t>0), \\
\tilde{u}_{i}(x, t) \geq \eta_{i}(x, t) \geq \widehat{u}_{i}(x, t) \quad\left(x \in \Omega, t \in I_{i}, i=1,2, \ldots, N\right),
\end{gathered}
$$


where inequality between vectors is in the componentwise sense. It is obvious that if $\tilde{U} \equiv$ $M \equiv\left(M_{1}, M_{2}, \ldots, M_{N}\right)$ and $\widehat{U} \equiv \delta \equiv\left(\delta_{1}, \delta_{2}, \ldots, \delta_{N}\right)$ are constant vectors, then the above inequalities become

$$
\begin{gathered}
f_{i}(x, M, M) \leq 0 \quad(x \in \Omega), \\
f_{i}(x, \delta, \delta) \geq 0 \quad(x \in \Omega), \\
M_{i} \geq \eta_{i}(x, t) \geq \delta_{i} \quad\left(x \in \Omega, t \in I_{i}\right), \quad i=1,2, \ldots, N .
\end{gathered}
$$

Notice that the boundary inequalities in (2.4) are trivially satisfied. For a given pair of constant vectors $M, \delta$ satisfying (2.5) we set

$$
S \equiv\{U \in C(\Omega): \delta \leq U \leq M\},
$$

where $C(\Omega)$ denotes the set of continuous functions on $\Omega$. In the following we give our basic hypotheses on $F(\cdot, U, V)$ with $V \equiv J * U$.

(H) The function $F(\cdot, U, V)$ is quasimonotone nondecreasing for $U, V \in S$, and for each $i, f_{i}(\cdot, U, V)$ satisfies the Lipschitz condition

$$
\left|f_{i}(x, U, V)-f_{i}\left(x, U^{\prime}, V^{\prime}\right)\right| \leq K_{i}\left(\left\|U-U^{\prime}\right\|+\left\|V-V^{\prime}\right\|\right) \quad\left(U, U^{\prime}, V, V^{\prime} \in S\right),
$$

where $K_{i}$ is a positive constant, and $\|w\|=\left|w_{1}\right|+\left|w_{2}\right|+\cdots+\left|w_{N}\right|$ for $w \in R^{N}(|\cdot|$ is a maximum norm).

It is clear that if there exists a positive constant vector $M=\left(M_{1}, M_{2}, \ldots, M_{N}\right)$ and a zero vector $o=(0,0, \ldots, 0)$ such that

$$
\begin{gathered}
f_{i}(x, M, M) \leq 0 \quad(x \in \Omega), \\
f_{i}(x, o, o) \geq 0 \quad(x \in \Omega), i=1,2, \ldots, N,
\end{gathered}
$$

then the pairs $\tilde{U}=M, \widehat{U}=o$ are coupled upper and lower solutions of (2.1) whenever $M_{i} \geq$ $\eta_{i}(x, t) \geq 0$. By an application of [12, Theorem 2.1] we have the following global existence result.

Lemma 2.3. Let $\tilde{U} \equiv M \equiv\left(M_{1}, M_{2}, \ldots, M_{N}\right), \widehat{U} \equiv \delta \equiv\left(\delta_{1}, \delta_{2}, \ldots, \delta_{N}\right)$ be a pair of constant vectors satisfying (2.5) and $M \geq \delta$, and let hypothesis $(\boldsymbol{H})$ hold. Then for any $\eta(x, t)$ in $S$, problem (2.1) has a unique global solution $U(x, t) \in S$ for all $t \geq 0$. In particular, if condition (2.8) holds for every $M \geq$ o then problem (2.1) has a unique bounded nonnegative global solution whenever $\eta(x, t) \geq 0$. 


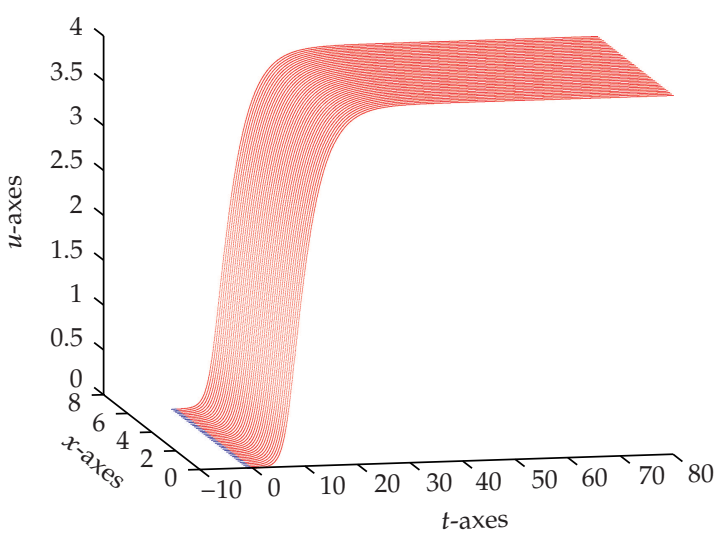

(a)

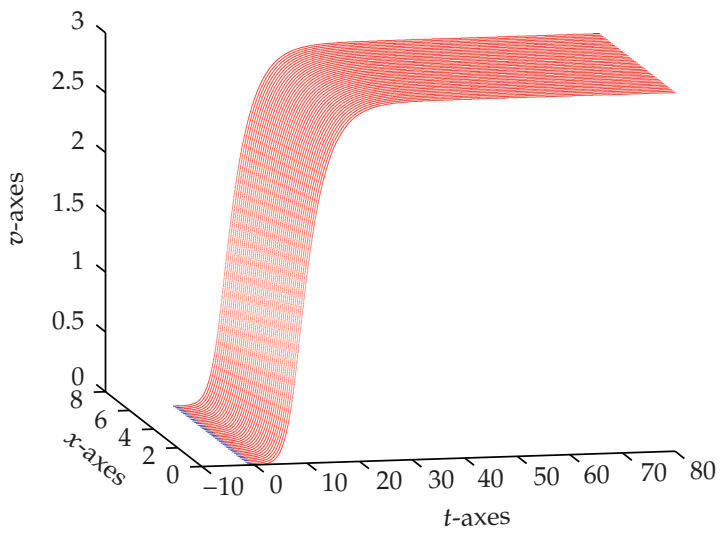

(b)

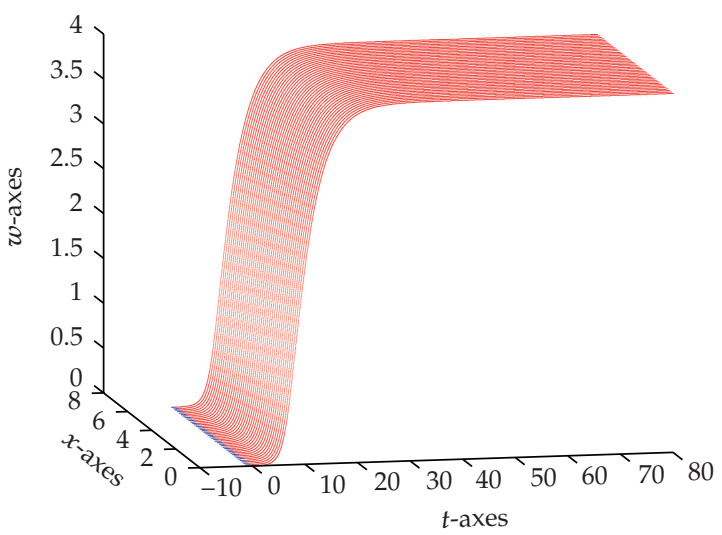

(c)

Figure 2: The solution of system (4.2). 
In system (2.1) if $f_{i}$ is given in the form

$$
f_{i}(x, U, V)=u_{i} f_{i}^{*}(x, U, V), \quad i=1,2, \ldots, N,
$$

then the pairs $\tilde{U}=M, \widehat{U}=o$ satisfy $(2.8)$ whenever

$$
f_{i}^{*}(x, M, M) \leq 0 \quad(x \in \Omega), i=1,2, \ldots, N
$$

Moreover, by the positivity lemma for the linear scalar parabolic problem

$$
\begin{gathered}
\frac{\partial W_{i}}{\partial t}-L_{i} W_{i}=W_{i} f_{i}^{*}(x, U, V) \quad(x \in \Omega, t>0), \\
\frac{\partial W_{i}}{\partial n}=0 \quad(x \in \partial \Omega, t>0) \\
W_{i}(x, 0)=\eta_{i}(x, 0) \quad x \in \Omega, i=1,2, \ldots, N
\end{gathered}
$$

where $U \equiv U(x, t)$ is the solution of $(2.1)$, the solution $W_{i} \equiv W_{i}(x, t)$ is positive in $(0,+\infty) \times \Omega$ whenever $\eta_{i}(x, 0) \geq 0$ and not identical 0 (cf. [1]). Since for each $i$ the solution component $u_{i} \equiv u_{i}(x, t)$ is also a solution of (2.11) the uniqueness property of $W_{i}$ ensures that $u_{i}(x, t)=$ $W_{i}(x, t)>0$ in $(0,+\infty) \times \Omega$. This observation leads to the following.

Lemma 2.4. Let $f_{i}(x, U, V)$ be given by (2.9) and satisfy hypothesis $(\boldsymbol{H})$, and let $M \equiv$ $\left(M_{1}, M_{2}, \ldots, M_{N}\right)$ be a constant vector satisfying (2.10). Then for any nontrivial nonnegative $\eta_{i}(x, t) \leq M_{i}$ problem (2.1) has a unique bounded positive solution $u(x, t)$ in $(0,+\infty) \times \Omega$.

To investigate the asymptotic behavior of the solution of (2.1), we consider the special case

$$
f_{i}(x, U, V)=g_{i}(U, V), \quad i=1,2, \ldots, N,
$$

where $g_{i}(U, V)$ is not explicitly dependent on $x$. The corresponding steady-state problem of (2.1) is given by

$$
\begin{gathered}
-L_{i} u_{i}=g_{i}(U, V) \quad(x \in \Omega), \\
\frac{\partial u_{i}}{\partial n}=0 \quad(x \in \partial \Omega,), i=1,2, \ldots, N
\end{gathered}
$$


Assume that $G(U, V) \equiv\left(g_{1}(U, V), \ldots, g_{N}(U, V)\right)$ is quasimonotone nondecreasing in $S$. Then by using $\bar{U}^{(0)}=M, \underline{U}^{(0)}=\delta$ as a pair of coupled initial iterations we construct two sequences $\left\{\bar{U}^{(m)}\right\} \equiv\left\{\bar{u}_{1}, \ldots, \bar{u}_{N}\right\},\left\{\underline{U}^{(m)}\right\} \equiv\left\{\underline{u}_{1}, \ldots, \underline{u}_{N}\right\}$ from the linear iteration process

$$
\begin{gathered}
-L_{i} \bar{u}_{i}^{(m)}+K_{i} \bar{u}_{i}^{(m)}=K_{i} \bar{u}_{i}^{(m-1)}+g_{i}\left(\bar{u}_{1}^{(m-1)}, \ldots, \bar{u}_{N}^{(m-1)}, J * \bar{u}_{1}^{(m-1)}, \ldots, J * \bar{u}_{N}^{(m-1)}\right), \\
-L_{i} \underline{u}_{i}^{(m)}+K_{i} \underline{u}_{i}^{(m)}=K_{i} \underline{u}_{i}^{(m-1)}+g_{i}\left(\underline{u}_{1}^{(m-1)}, \ldots, \underline{u}_{N}^{(m-1)}, J * \underline{u}_{1}^{(m-1)}, \ldots, J * \underline{u}_{N}^{(m-1)}\right), \\
\frac{\partial \bar{u}_{i}^{(m)}}{\partial n}=\frac{\partial \underline{u}_{i}^{(m)}}{\partial n}=0 \quad(i=1,2, \ldots, N),
\end{gathered}
$$

where $K_{i}$ is the Lipschitz constant in (2.7). It is clear that the sequences $\left\{\bar{U}^{(m)}\right\},\left\{\underline{U}^{(m)}\right\}$ are well defined. Since the initial iterations in (2.14) are the constant vectors $M, \delta$ and $g_{i}(U, V)$ is independent of $x$ whenever $U$ and $V$ are constants we conclude from the uniqueness property of solution for linear boundary-value problems that the solutions $\bar{u}_{i}^{(m)}, \underline{u}_{i}^{(m)}$ of (2.14) are constants and are given by

$$
\begin{aligned}
& \bar{u}_{i}^{(m)}=\bar{u}_{i}^{(m-1)}+\frac{1}{K_{i}} g_{i}\left(\bar{u}_{1}^{(m-1)}, \ldots, \bar{u}_{N}^{(m-1)}, \bar{u}_{1}^{(m-1)}, \ldots, \bar{u}_{N}^{(m-1)}\right), \\
& \underline{u}_{i}^{(m)}=\underline{u}_{i}^{(m-1)}+\frac{1}{K_{i}} g_{i}\left(\underline{u}_{1}^{(m-1)}, \ldots, \underline{u}_{N}^{(m-1)}, \underline{u}_{1}^{(m-1)}, \ldots, \underline{u}_{N}^{(m-1)}\right) \quad(i=1,2, \ldots, N) .
\end{aligned}
$$

It is easy to show from the quasimonotone nondecreasing property of $G(U, V)$ that the sequences $\bar{u}_{i}^{(m)}, \underline{u}_{i}^{(m)}$ possess the monotone property

$$
\delta \leq \underline{U}^{(m)} \leq \underline{U}^{(m+1)} \leq \bar{U}^{(m+1)} \leq \bar{U}^{(m)} \leq M \quad(m=1,2, \ldots)
$$

(cf. [12]). The above property implies that the constant limits

$$
\lim _{m \rightarrow \infty} \bar{U}^{(m)}=\bar{\rho}, \quad \lim _{m \rightarrow \infty} \underline{U}^{(m)}=\underline{\rho}
$$

exist and satisfy the relation $\delta \leq \underline{\rho} \leq \bar{\rho} \leq$. Letting $m \rightarrow \infty$ in (2.15) shows that $\rho$ and $\bar{\rho}$ satisfy the equations

$$
g_{i}(\bar{\rho}, \bar{\rho})=0, \quad g_{i}(\underline{\rho}, \underline{\rho})=0 \quad(i=1,2, \ldots, N)
$$

It is easy to verify that the limits $\bar{\rho}$ and $\rho$ are maximal and minimal solutions of systems (2.13) in $S$, respectively, furthermore, if $\bar{\rho}^{-}=\rho\left(\equiv \rho^{*}\right)$ then $\rho^{*}$ is the unique solution in $S$ (cf. $[1,12])$. In the latter case, the time-dependent solution of (2.1) (with $f_{i}(x, U, V)$ given by (2.12)) converges to $\rho^{*}$ as $t \rightarrow \infty$ (cf. [12]). To summarize the above conclusions we have the following. 
Lemma 2.5. Let the conditions in Lemma 2.3 be satisfied with respect to the function given by (2.12). Then

(i) the sequences $\left\{\bar{U}^{(m)}\right\},\left\{\underline{U}^{(m)}\right\}$ given by $(2.14)$ with $\bar{U}^{(0)}=M, \underline{U}^{(0)}=\delta$ are constant functions and converge monotonically to their respective constant limits $\bar{\rho}$ and $\rho$ that satisfy (2.18);

(ii) for any $m \in N$, if $\underline{U}^{(m)} \leq \eta(x, t) \leq \bar{U}^{(m)}$, then the constant pairs $\tilde{U}=\bar{U}^{(m)}, \widehat{U}=\underline{U}^{(m)}$ are also coupled upper and lower solutions of (2.1), and the problem (2.1) has a unique positive solution $U(x, t)$ such that $\underline{U}^{(m)} \leq U(x, t) \leq \bar{U}^{(m)}$ and $\rho \leq u(x, t) \leq \bar{\rho}$, as $t \rightarrow$ $\infty \quad(x \in \bar{\Omega})$;

(iii) if $\bar{\rho}=\rho\left(\equiv \rho^{*}\right)$ then $\rho^{*}$ is the unique solution of (2.13) in $S$, and for any initial function $\eta(x, t) \in S$ the corresponding solution $U(x, t)$ of $(2.1)$ converges to $\rho^{*}$ as $t \rightarrow \infty$.

In Lemma 2.5 the convergence of the time-dependent solution $U(x, t)$ to $\rho^{*}$ is for the class of initial functions in $S$. For arbitrary nontrivial nonnegative initial functions we have the following result from [12].

Lemma 2.6. Let the conditions in Lemma 2.5 be satisfied, and let $U \equiv\left(u_{1}, u_{2}, \ldots, u_{N}\right)$ be the solution of (2.1) corresponding to an arbitrary nontrivial nonnegative $\eta(x, t)$. Assume that $\bar{\rho}=\rho\left(\equiv \rho^{*}\right)$ and there exists $t^{*}>0$ such that

$$
\delta_{i} \leq u_{i}(x, t) \leq M_{i} \quad \text { for } t^{*}-\tau_{i} \leq t \leq t^{*}, x \in \bar{\Omega},(i=1,2, \ldots, N) .
$$

Then $U(x, t)$ converges to $\rho^{*}$ as $t \rightarrow \infty$.

To prove the existence and uniqueness of constant positive solution of the steady-state problem (1.13) we also need the following result.

Lemma 2.7. Let $a_{i}>0, b_{i}>0, c_{i}>0(i=1,2,3)$. If $a_{1} b_{2} c_{3}-a_{2} b_{1} c_{3}-a_{1} b_{3} c_{2}>0$ then the inequalities

$$
\begin{gathered}
a_{1} x-b_{1} y>0, \\
-a_{2} x+b_{2} y-c_{2} z>0, \\
-b_{3} y+c_{3} z>0
\end{gathered}
$$

have a positive solution $\left(\rho_{1}, \rho_{2}, \rho_{3}\right)$.

Proof. With the inequalities (2.20) exists a positive solution if and only if there exist positive constants $\lambda_{1}, \lambda_{2}, \lambda_{3}$ such that the following equations:

$$
\begin{gathered}
a_{1} x-b_{1} y=\lambda_{1}, \\
-a_{2} x+b_{2} y-c_{2} z=\lambda_{2}, \\
-b_{3} y+c_{3} z=\lambda_{3}
\end{gathered}
$$


have a positive solution. Let $A=\left(\begin{array}{ccc}a_{1} & -b_{1} & 0 \\ -a_{2} & b_{2} & -c_{2} \\ 0 & -b_{3} & c_{3}\end{array}\right)$, we have $|A|>0$. From the Cramer theorem, the solutions of (2.21) are as follows:

$$
\begin{aligned}
& x=\frac{\lambda_{1}\left|\begin{array}{rr}
b_{2} & -c_{2} \\
-b_{3} & c_{3}
\end{array}\right|-\lambda_{2}\left|\begin{array}{cc}
-b_{1} & 0 \\
-b_{3} & c_{3}
\end{array}\right|+\lambda_{3}\left|\begin{array}{cc}
-b_{1} & 0 \\
b_{2} & -c_{2}
\end{array}\right|}{|A|} \\
& =\frac{\lambda_{1}\left(b_{2} c_{3}-b_{3} c_{2}\right)+\lambda_{2} b_{1} c_{3}+\lambda_{3} b_{1} c_{3}}{|A|}, \\
& y=\frac{-\lambda_{1}\left|\begin{array}{cc}
-a_{2} & -c_{2} \\
0 & c_{3}
\end{array}\right|+\lambda_{2}\left|\begin{array}{cc}
a_{1} & 0 \\
0 & c_{3}
\end{array}\right|-\lambda_{3}\left|\begin{array}{cc}
a_{1} & 0 \\
-a_{2} & -c_{2}
\end{array}\right|}{|A|} \\
& =\frac{\lambda_{1} a_{2} c_{3}+\lambda_{2} a_{1} c_{3}+\lambda_{3} a_{1} c_{2}}{|A|}, \\
& z=\frac{\lambda_{1}\left|\begin{array}{cc}
-a_{2} & b_{2} \\
0 & -b c_{3}
\end{array}\right|-\lambda_{2}\left|\begin{array}{cc}
a_{1} & -b_{1} \\
0 & -b_{3}
\end{array}\right|+\lambda_{3}\left|\begin{array}{ll}
a_{1} & -b_{1} \\
0 & -b_{3}
\end{array}\right|}{|A|} \\
& =\frac{\lambda_{1} a_{2} b_{3}+\lambda_{2} a_{1} b_{3}+\lambda_{3}\left(a_{1} b_{2}-a_{2} b_{1}\right)}{|A|} .
\end{aligned}
$$

Let $\lambda_{1}=\lambda_{3}=1, \lambda_{2}=\max \left\{\left(1-b_{1} c_{3}-b_{2} c_{3}+b_{3} c_{2}\right) / b_{1} c_{3},\left(1-a_{1} b_{2}+a_{2} b_{1}-a_{2} b_{3}\right) / a_{1} b_{3}, 1\right\}$, then with the equations (2.21) exists a positive solution $\left(\rho_{1}, \rho_{2}, \rho_{3}\right)$. Therefore the inequalities (2.20) have a positive solution.

\section{The Main Theorems and Proof}

To prove the main results in this paper, we apply Lemmas 2.3-2.7 for the models in (1.1)(1.2) with $U=(u, v, w)$ by constructing a suitable pair of constant upper and lower solutions $\tilde{U}=M$ and $\widehat{U}=\delta$. This is equivalent to show that $M$ and $\delta$ satisfy condition (2.5) for the reaction functions $f_{i}(x, U, V)$.

Theorem 3.1. Let $e_{i}^{*} \equiv e_{i}+\beta_{i}>0(i=1,2,3)$ and $b_{2}^{*} \equiv b_{2}+\alpha_{2}>0$. If $c_{1} c_{2} c_{3}-c_{1} e_{2}^{*} e_{3}^{*}-e_{1}^{*} b_{2}^{*} c_{3}>0$, then the following statements hold true.

(i) The steady-state problem (1.9) has a unique constant positive solution.

(ii) For any nontrivial nonnegative $\left(\eta_{1}, \eta_{2}, \eta_{3}\right)$, problems (1.1)-(1.2) have a unique nonnegative global solution $(u, v, w)$, and the solution is uniformly boundary in $\bar{\Omega} \times[0, \infty)$.

(iii) The positive constant solution $\rho^{*}$ of the steady-state problem (1.9) is globally asymptotic stable. 


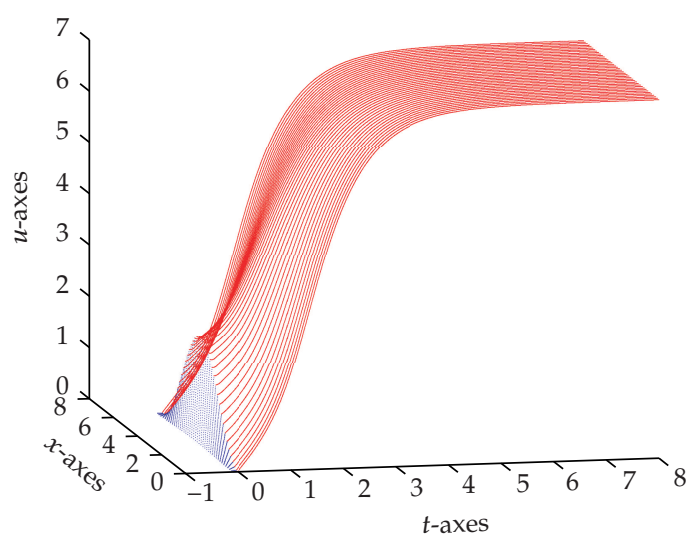

(a)

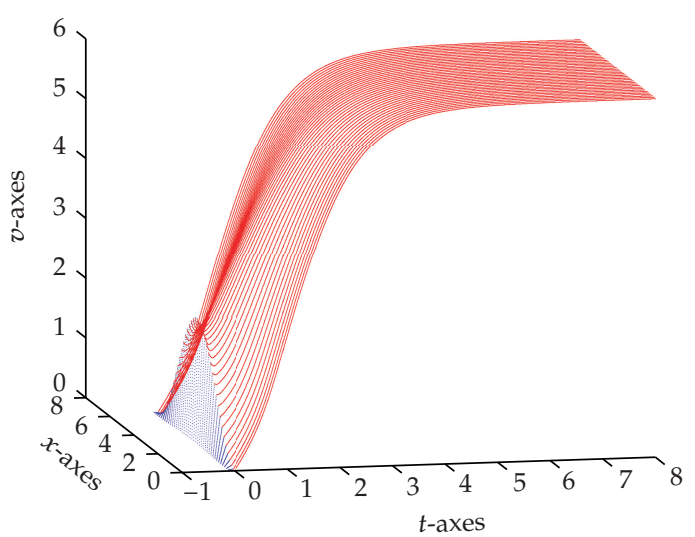

(b)

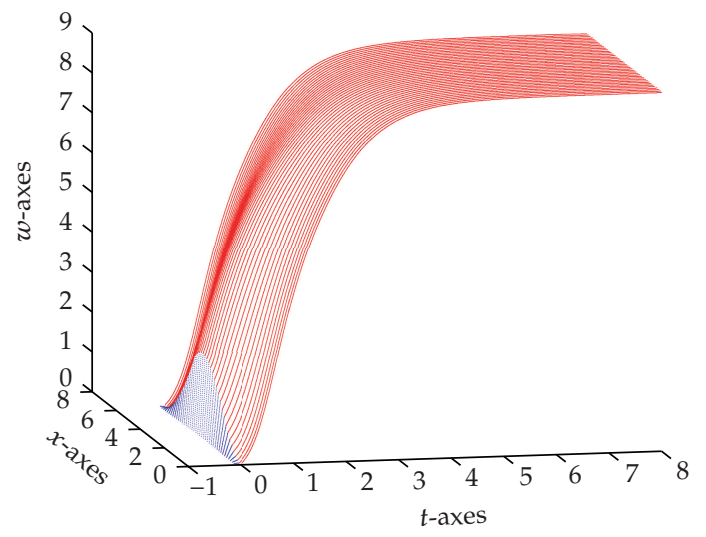

(c)

Figure 3: The solution of system (4.3).

Proof. (i)Since the boundary conditions are Neumann type, we seek a pair of positive constant vectors $M=\left(M_{1}, M_{2}, M_{3}\right), \delta=\left(\delta_{1}, \delta_{2}, \delta_{3}\right)$ satisfying

$$
\begin{gathered}
M_{1}\left(a_{1}-c_{1} M_{1}+e_{1}^{*} M_{2}\right) \leq 0 \leq \delta_{1}\left(a_{1}-c_{1} \delta_{1}+e_{1}^{*} \delta_{2}\right) \\
M_{2}\left(a_{2}+b_{2}^{*} M_{1}-c_{2} M_{2}+e_{2}^{*} M_{3}\right) \leq 0 \leq \delta_{2}\left(a_{2}+b_{2}^{*} \delta_{1}-c_{2} \delta_{2}+e_{2}^{*} \delta_{3}\right), \\
M_{3}\left(a_{3}+e_{3}^{*} M_{2}-c_{3} M_{3}\right) \leq 0 \leq \delta_{3}\left(a_{3}+e_{3}^{*} \delta_{2}-c_{3} \delta_{3}\right) \\
\frac{\partial \delta_{i}}{\partial n} \leq 0 \leq \frac{\partial M_{i}}{\partial n} \quad(i=1,2,3) .
\end{gathered}
$$




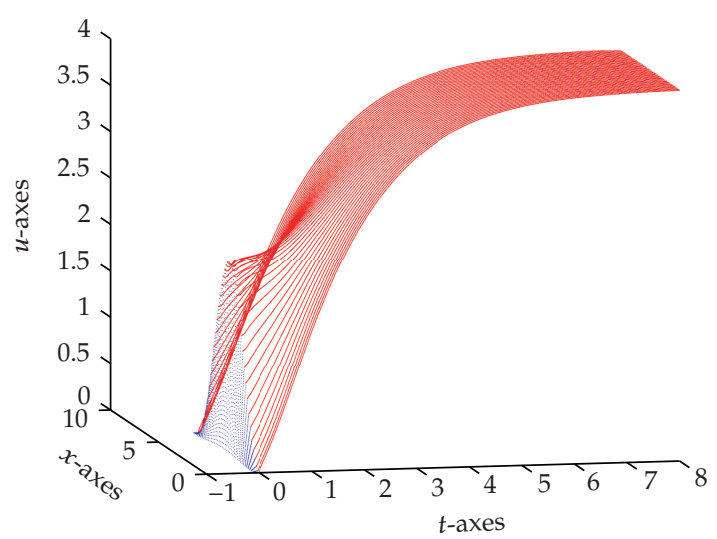

(a)

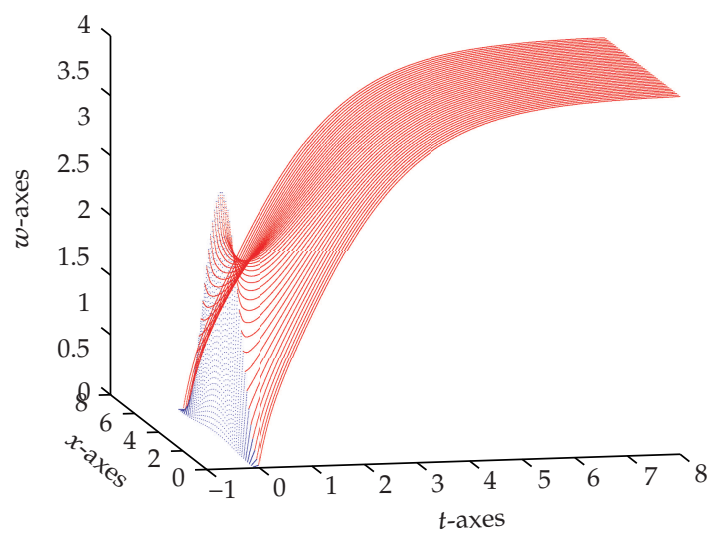

(b)

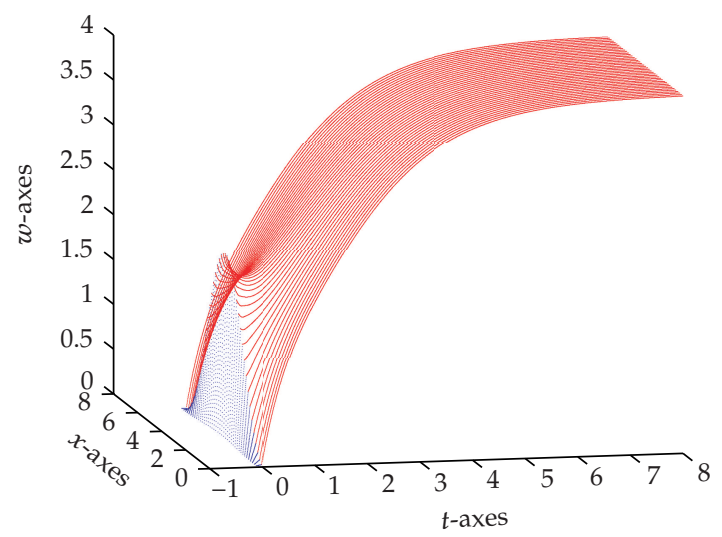

(c)

Figure 4: The solution of system (4.4). 
It is clear that the boundary conditions are satisfied, and from the positive property of $M_{i}$ and $\delta_{i}$ that condition (3.1) is satisfied if

$$
\begin{gathered}
\left(a_{1}-c_{1} M_{1}+e_{1}^{*} M_{2}\right) \leq 0 \leq\left(a_{1}-c_{1} \delta_{1}+e_{1}^{*} \delta_{2}\right), \\
\left(a_{2}+b_{2}^{*} M_{1}-c_{2} M_{2}+e_{2}^{*} M_{3}\right) \leq 0 \leq\left(a_{2}+b_{2}^{*} \delta_{1}-c_{2} \delta_{2}+e_{2}^{*} \delta_{3}\right), \\
\left(a_{3}+e_{3}^{*} M_{2}-c_{3} M_{3}\right) \leq 0 \leq\left(a_{3}+e_{3}^{*} \delta_{2}-c_{3} \delta_{3}\right) .
\end{gathered}
$$

We construct the following inequalities:

$$
\begin{gathered}
c_{1} x-e_{1}^{*} y>0, \\
-b_{2}^{*} x+c_{2} y-e_{2}^{*} z>0, \\
-e_{3}^{*} y+c_{3} z>0 .
\end{gathered}
$$

Let $A=\left(\begin{array}{ccc}c_{1} & -e_{1}^{*} & 0 \\ -b_{2}^{*} & c_{2} & -e_{2}^{*} \\ 0 & -e_{3}^{*} & c_{3}\end{array}\right)$, we have $|A|>0$. Since $e_{i}^{*} \equiv e_{i}+\beta_{i}>0 \quad(i=1,2,3)$ and $b_{2}^{*} \equiv b_{2}+\alpha_{2}>0$, from the Lemma 2.7 the inequalities (3.3) have a positive solution $\left(\lambda_{1}, \lambda_{2}, \lambda_{3}\right)$. By choosing $M=\left(M_{1}, M_{2}, M_{3}\right)=\left(P \lambda_{1}, P \lambda_{2}, P \lambda_{3}\right), \delta=\left(\delta_{1}, \delta_{2}, \delta_{3}\right)=\left(\gamma \lambda_{1}, \gamma \lambda_{2}, \gamma \lambda_{3}\right)$, where $P$ and $\gamma$ are a sufficiently large and small positive constant, respectively, we see that all the inequalities in (3.2) are satisfied. That is, $M$ and $\delta$ is a pair of positive constant upper-lower solution of (1.1)-(1.2). By Lemma 2.5 the sequences governed by (2.14) with the reaction function $\left(f_{1}, f_{2}, f_{3}\right)$ given by (1.1) converge monotonically, respectively, to some constant limits $\bar{\rho} \equiv$ $\left(\bar{\rho}_{1}, \bar{\rho}_{2}, \bar{\rho}_{3}\right), \underline{\rho} \equiv\left(\underline{\rho}_{1}, \underline{\rho}_{2}, \underline{\rho}_{3}\right)$ that satisfy

$$
\begin{aligned}
\bar{\rho}_{1}\left(a_{1}-c_{1} \bar{\rho}_{1}+e_{1}^{*} \bar{\rho}_{2}\right) & =0, & & \underline{\rho}_{1}\left(a_{1}-c_{1} \underline{\rho}_{1}+e_{1}^{*} \underline{\rho}_{2}\right)=0, \\
\bar{\rho}_{2}\left(a_{2}+b_{2}^{*} \bar{\rho}_{1}-c_{2} \bar{\rho}_{2}+e_{2}^{*} \bar{\rho}_{3}\right) & =0, & & \underline{\rho}_{2}\left(a_{2}+b_{2}^{*} \underline{\rho}_{1}-c_{2} \underline{\rho}_{2}+e_{2}^{*} \underline{\rho}_{3}\right)=0, \\
\bar{\rho}_{3}\left(a_{3}+e_{3}^{*} \bar{\rho}_{2}-c_{3} \bar{\rho}_{3}\right) & =0, & & \underline{\rho}_{3}\left(a_{3}+e_{3}^{*} \underline{\rho}_{2}-c_{3} \underline{\rho}_{3}\right)=0 .
\end{aligned}
$$

Let $\rho_{i}=\bar{\rho}_{i}-\underline{\rho}_{i}(i=1,2,3)$. Then by the positivity of $\bar{\rho}_{i}$ and $\underline{\rho}_{i}$, a subtraction of the above pairs of equations, leads to

$$
\begin{gathered}
c_{1} \rho_{1}-e_{1}^{*} \rho_{2}=0, \\
-b_{2}^{*} \rho_{1}+c_{2} \rho_{2}-e_{2}^{*} \rho_{3}=0, \\
-e_{3}^{*} \rho_{2}+c_{3} \rho_{3}=0 .
\end{gathered}
$$

Since these equations are equivalent to $A \rho=0$, where $A$ is coefficient matrix and $\rho$ is the column vector $\left(\rho_{1}, \rho_{2}, \rho_{3}\right)^{T}$, we conclude from the nonsingular property of $A$ that $\rho=o$. This proves $\bar{\rho}=\rho\left(\equiv \rho^{*}\right)$ and $\rho^{*}$ is the unique positive solution of (1.13) in $S$. 
(ii)It is obvious that the pairs $M=\left(M_{1}, M_{2}, M_{3}\right)=\left(P \lambda_{1}, P \lambda_{2}, P \lambda_{3}\right), \delta=\left(\delta_{1}, \delta_{2}, \delta_{3}\right)=$ $(0,0,0)$ satisfy all the equalities in (3.1). By Lemma 2.3 and the arbitrariness of $P$, problems (1.1)-(1.2) have a unique bounded nonnegative global solution $(u, v, w)$ for any nontrivial nonnegative $\left(\eta_{-} 1, \eta-2, \eta_{-} 3\right)$.

(iii)Lemma 2.5 ensures that for any initial function $\eta(x, t) \equiv\left(\eta_{1}(x, t), \eta_{2}(x, t), \eta_{3}(x, t)\right)$ with $\gamma\left(\lambda_{1}, \lambda_{2}, \lambda_{3}\right) \equiv \delta \leq \eta(x, t) \leq M \equiv P\left(\lambda_{1}, \lambda_{2}, \lambda_{3}\right)$, where $P$ and $\gamma$ are a sufficiently large and small positiveconstants, respectively, the corresponding solution $U(x, t) \equiv(u(x, t), v(x, t), w(x, t))$ of (1.1)-(1.2) converges to $\rho^{*}$ as $t \rightarrow \infty$. To show this convergence property for an arbitrary nontrivial nonnegative $\eta(x, t)$, we observe from Lemma 2.4, the arbitrary smallness of $\gamma$ and the arbitrary largeness of $P$ that there exists $t_{0}>0$ such that $\delta \leq U(x, t) \leq M$ on $\left[t_{0}, \infty\right) \times \bar{\Omega}$. The upper and lower bounds of $U(x, t)$ show that there exists $t^{*}>0$ such that condition (2.19) is satisfied for $U \equiv\left(u_{1}, u_{2}, u_{3}\right)=(u, v, w)$. The conclusion of $U(x, t) \rightarrow \rho^{*}$ as $t \rightarrow$ $\infty$ follows from Lemma 2.6. We next consider the stability of $U(x, t)$. We observe from Lemma 2.5 that for any $U\left(\rho^{*}, \varepsilon\right)$ there exists $\delta>0$ such that $U(x, t) \in U^{*} \equiv$ $U\left(\rho^{*}, \delta\right) \subseteq\left(\underline{U}^{(m)}, \bar{U}^{(m)}\right) \subset U\left(\rho^{*}, \varepsilon\right)$ as initial function $\eta(x, t) \in U^{*}$. This completes the proof.

Corollary 3.2. All the results of Theorem 3.1 hold true if $d_{i}=0$ for some or all $i$. In particular, these results hold true for the ordinary differential system (1.8). They are also true if the reaction functions involve no time delays.

Proof. This follows from the argument in the proof of Theorem 3.1 by letting $d_{i}=0$ (and without the corresponding boundary condition) and by letting $\tau_{i}=0(i=1,2,3)$, respectively.

Remark 3.3. Our model and result are different from the existence ones such as those of Pao [29] and Kim and Lin [22, 23]. In some sense, we enrich the results of the 3-specics LotkaVolterra reaction-diffusion systems.

\section{Numerical Simulations}

In this section, we give numerical simulations supporting our theoretical analysis. As an example, we consider system (1.1) with different diffusion rates $d_{i}$, birth rate $a_{i}$, and time delays $\tau_{i}$, that is, the following systems:

$$
\begin{aligned}
\frac{\partial u}{\partial t}-u_{x x}=u(t, x) & {\left[1-u(t, x)+\frac{1}{2} v(t, x)+\frac{1}{2} v(t-0.1, x)\right] \quad(2 \pi>x>0, t>0) } \\
\frac{\partial v}{\partial t}-v_{x x}=v(t, x) & {\left[1+\frac{1}{2} u(t, x)-3 v(t, x)+\frac{1}{2} w(t, x)\right.} \\
& \left.+\frac{1}{2} u(t-0.1, x)+\frac{1}{2} w(t-0.1, x)\right] \quad(2 \pi>x>0, t>0) \\
\frac{\partial w}{\partial t}-w_{x x}=w(t, x) & {\left[1+\frac{1}{2} v(t, x)-w+\left(\frac{1}{2} v(t-0.1, x)\right)\right] \quad(2 \pi>x>0, t>0) }
\end{aligned}
$$




$$
\begin{aligned}
& \frac{\partial u}{\partial n}=\frac{\partial v}{\partial n}=\frac{\partial w}{\partial n}=0 \quad(t>0, x=0,2 \pi), \\
& u(t, x)=v(t, x)=w(t, x)=(1+10 t)\left[1-\sin \left(x+\frac{\pi}{2}\right)\right] \quad(t, x) \in[-0.1,0] \times[0,2 \pi] . \\
& \frac{\partial u}{\partial t}-u_{x x}=u(t, x)\left[1-u(t, x)+\frac{1}{2} v(t, x)+\frac{1}{2} v(t-0.01, x)\right] \quad(2 \pi>x>0, t>0), \\
& \frac{\partial v}{\partial t}-v_{x x}=v(t, x)\left[1+\frac{1}{2} u(t, x)-3 v(t, x)+\frac{1}{2} w(t, x),\right. \\
& \left.+\frac{1}{2} u(t-0.01, x)+\frac{1}{2} w(t-0.01, x)\right] \quad(2 \pi>x>0, t>0), \\
& \frac{\partial w}{\partial t}-w_{x x}=w(t, x)\left[1+\frac{1}{2} v(t, x)-w+\frac{1}{2} v(t-0.01, x)\right] \quad(2 \pi>x>0, t>0), \\
& \frac{\partial u}{\partial n}=\frac{\partial v}{\partial n}=\frac{\partial w}{\partial n}=0 \quad(t>0, x=0,2 \pi), \\
& u(t, x)=v(t, x)=w(t, x)=(1+t)\left[1-\sin \left(x+\frac{\pi}{2}\right)\right] \quad(t, x) \in[-0.01,0] \times[0,2 \pi] . \\
& \frac{\partial u}{\partial t}-u_{x x}=u(t, x)\left[1-u(t, x)+\frac{1}{2} v(t, x)+\frac{1}{2} v(t-0.1, x)\right] \quad(2 \pi>x>0, t>0), \\
& \frac{\partial v}{\partial t}-v_{x x}=v(t, x)\left[2+\frac{1}{2} u(t, x)-3 v(t, x)+\frac{1}{2} w(t, x),\right. \\
& \left.+\frac{1}{2} u(t-0.1, x)+\frac{1}{2} w(t-0.1, x)\right] \quad(2 \pi>x>0, t>0), \\
& \frac{\partial w}{\partial t}-w_{x x}=w(t, x)\left[3+\frac{1}{2} v(t, x)-w+\frac{1}{2} v(t-0.1, x)\right] \quad(2 \pi>x>0, t>0), \\
& \frac{\partial u}{\partial n}=\frac{\partial v}{\partial n}=\frac{\partial w}{\partial n}=0 \quad(t>0, x=0,2 \pi), \\
& u(t, x)=v(t, x)=w(t, x)=(1+10 t)\left[1-\sin \left(x+\frac{\pi}{2}\right)\right] \quad(t, x) \in[-0.1,0] \times[0,2 \pi] . \\
& \frac{\partial u}{\partial t}-u_{x x}=u(t, x)\left[1-u(t, x)+\frac{1}{2} v(t, x)+\frac{1}{2} v(t-0.1, x)\right] \quad(2 \pi>x>0, t>0), \\
& \frac{\partial v}{\partial t}-2 v_{x x}=v(t, x)\left[1+\frac{1}{2} u(t, x)-3 v(t, x)+\frac{1}{2} w(t, x),\right. \\
& \left.+\frac{1}{2} u(t-0.1, x)+\frac{1}{2} w(t-0.1, x)\right] \quad(2 \pi>x>0, t>0), \\
& \frac{\partial w}{\partial t}-3 w_{x x}=w(t, x)\left[1+\frac{1}{2} v(t, x)-w+\frac{1}{2} v(t-0.1, x)\right] \quad(2 \pi>x>0, t>0), \\
& \frac{\partial u}{\partial n}=\frac{\partial v}{\partial n}=\frac{\partial w}{\partial n}=0 \quad(t>0, x=0,2 \pi), \\
& u(t, x)=v(t, x)=w(t, x)=(1+10 t)\left[1-\sin \left(x+\frac{\pi}{2}\right)\right] \quad(t, x) \in[-0.1,0] \times[0,2 \pi] .
\end{aligned}
$$


By using the classical implicit format solving the partial differential equations and the method of steps for differential difference equations and employing the software package MATLAB7.0, we can solve the numerical solutions of systems (4.1), (4.2), (4.3), and (4.4) which are shown respectively in Figures 1, 2, 3, and 4.

\section{Conclusions}

It is well known that the analysis of stability for a system of delay reaction-diffusion multispecies model is quite difficult since the reaction among multispecies is more complex. Therefore, the works on this subject are very rare. A detailed analysis on the stability for a two-prey one-predator model, one-prey two-predator model, and three-species food-chain model with delay and diffusion was given by Pao [29], and he obtained some simple and easily verifiable conditions for the existence and global asymptotic stability of a positive steady-state solution for each of the three model problems.

In this paper, based on the ideas of Pao [12], we have considered a delay cooperative three-species system with Neumann boundary condition. It is shown that the system has a positive equilibrium under some certain conditions. We have obtained the similar conclusions to those of Pao [29]. More precisely, we have obtained the following results.

(a) The steady-state problem (1.9) has a unique constant positive solution if $c_{1} c_{2} c_{3}-$ $c_{1} e_{2}^{*} e_{3}^{*}-e_{1}^{*} b_{2}^{*} c_{3}>0$.

(b) For any nontrivial nonnegativ $\left(\eta_{1}, \eta_{2}, \eta_{3}\right)$, problems (1.1)-(1.2) have a unique nonnegative global solution $(u, v, w)$, and the solution is uniformly boundary in $\bar{\Omega} \times[0, \infty)$.

(c) The positive constant solution $\rho^{*}$ of the steady-state problem (1.9) is globally asymptotic stable.

The condition $c_{1} c_{2} c_{3}-c_{1} e_{2}^{*} e_{3}^{*}-e_{1}^{*} b_{2}^{*} c_{3}>0$ involves only the reaction rate constants, which shows that the diffusion rates $d_{i}$, the birth rate $a_{i}$, and time delays $\tau_{i}$ do not bring effect on permanence of one species as well as contribution to its extinction. The result of global asymptotic stability implies that the three-species model system coexists, is permanent, and the trivial and all semitrivial solutions are unstable.

\section{Acknowledgments}

The authors are grateful to the referee for her/his comments. This work is supported by Science and Technology Study Project of Chongqing Municipal Education Commission (Grant no. KJ 080511) of China, Natural Science Foundation Project of CQ CSTC (Grant no. 2008BB7415 and 2007BB2450) of China, Foundation Project of Doctor Graduate Student Innovation of Beijing University of Technology of China, the NSFC (Grant nos.10471009), and BSFC (Grant no. 1052001) of China.

\section{References}

[1] C. V. Pao, Nonlinear Parabolic and Elliptic Equations, Plenum, New York, NY, USA, 1992.

[2] B. S. Goh, "Stability in models of mutualism," The American Naturalist, vol. 113, no. 2, pp. 261-275, 1979.

[3] R. S. Cantrell, C. Cosner, and V. Hutson, "Permanence in ecological systems with spatial heterogeneity," Proceedings of the Royal Society of Edinburgh. Section A, vol. 123, no. 3, pp. 533-559, 1993. 
[4] C. Cosner and A. C. Lazer, "Stable coexistence states in the Volterra-Lotka competition model with diffusion," SIAM Journal on Applied Mathematics, vol. 44, no. 6, pp. 1112-1132, 1984.

[5] P. Korman and A. Leung, "On the existence and uniqueness of positive steady states in the VolterraLotka ecological models with diffusion," Applicable Analysis, vol. 26, no. 2, pp. 145-160, 1987.

[6] A. Leung and D. Clark, "Bifurcations and large-time aysmptotic behavior for prey-predator reactiondiffusion equations with Dirichlet boundary data," Journal of Differential Equations, vol. 35, no. 1, pp. 113-127, 1980.

[7] W. Ruan and W. Feng, "On the fixed point index and multiple steady-state solutions of reactiondiffusion systems," Differential and Integral Equations, vol. 8, no. 2, pp. 371-391, 1995.

[8] Y. Yamada, "Stability of steady states for prey-predator diffusion equations with homogeneous Dirichlet conditions," SIAM Journal on Mathematical Analysis, vol. 21, no. 2, pp. 327-345, 1990.

[9] L. Zhou and C. V. Pao, "Asymptotic behavior of a competition-diffusion system in population dynamics," Nonlinear Analysis, vol. 6, no. 11, pp. 1163-1184, 1982.

[10] Y. Xia and M. Lin, "Existence of positive periodic solution of mutualism system with infinite delays," Annals of Differential Equations, vol. 21, no. 3, pp. 448-453, 2005.

[11] F. Yang and D. Ying, "Existence of positive solution of multidelays facultative mutualism system," Journal of Engineering Mathematics, vol. 3, pp. 64-68, 2002.

[12] C. V. Pao, "Convergence of solutions of reaction-diffusion systems with time delays," Nonlinear Analysis: Theory, Methods \& Applications, vol. 48, no. 3, pp. 349-362, 2002.

[13] Z. Y. Li, "Asymptotic behavior of solutions of a cooperative-competitive reaction-diffusion equation," Acta Mathematicae Applicatae Sinica, vol. 7, no. 4, pp. 437-450, 1984.

[14] W. Feng and X. Lu, "Some coexistence and extinction results for a 3-species ecological system," Differential and Integral Equations, vol. 8, no. 3, pp. 617-626, 1995.

[15] X. He and K. Gopalsamy, "Persistence, attractivity, and delay in facultative mutualism," Journal of Mathematical Analysis and Applications, vol. 215, no. 1, pp. 154-173, 1997.

[16] S. N. Zheng, "A reaction-diffusion system of a competitor-competitor-mutualist model," Journal of Mathematical Analysis and Applications, vol. 124, no. 1, pp. 254-280, 1987.

[17] S. Fu and S. Cui, "Persistence in a periodic competitor-competitor-mutualist diffusion system," Journal of Mathematical Analysis and Applications, vol. 263, no. 1, pp. 234-245, 2001.

[18] W. Feng, "Coexistence, stability, and limiting behavior in a one-predator-two-prey model," Journal of Mathematical Analysis and Applications, vol. 179, no. 2, pp. 592-609, 1993.

[19] N. Lakoš, "Existence of steady-state solutions for a one-predator-two-prey system," SIAM Journal on Mathematical Analysis, vol. 21, no. 3, pp. 647-659, 1990.

[20] A. Leung, "A study of three species prey-predator reaction-diffusions by monotone schemes," Journal of Mathematical Analysis and Applications, vol. 100, no. 2, pp. 583-604, 1984.

[21] C. V. Pao, "Dynamics of nonlinear parabolic systems with time delays," Journal of Mathematical Analysis and Applications, vol. 198, no. 3, pp. 751-779, 1996.

[22] K. Kim and Z. Lin, "Blowup in a three-species cooperating model," Applied Mathematics Letters, vol. 17, no. 1, pp. 89-94, 2004.

[23] K. Kim and Z. Lin, "Blowup estimates for a parabolic system in a three-species cooperating model," Journal of Mathematical Analysis and Applications, vol. 293, no. 2, pp. 663-676, 2004.

[24] P. Fergola, E. Beretta, and M. Cerasuolo, "Some new results on an allelopathic competition model with quorum sensing and delayed toxicant production," Nonlinear Analysis: Real World Applications, vol. 7, no. 5, pp. 1081-1095, 2006.

[25] Y. Kuang, Delay Differential Equations with Applications in Population Dynamics, vol. 191 of Mathematics in Science and Engineering, Academic Press, San Diego, Calif, USA, 1993.

[26] A. W. Leung, Systems of Nonlinear Partial Differential Equations, Mathematics and Its Applications, Kluwer Academic Publishers, Dordrecht, The Netherlands, 1989.

[27] Y. Takeuchi, Global Dynamical Properties of Lotka-Volterra Systems, World Scientific, Singapore, 1996.

[28] J. Wu, Theory and Applications of Partial Functional-Differential Equations, vol. 119 of Applied Mathematical Sciences, Springer, New York, NY, USA, 1996.

[29] C. V. Pao, "Global asymptotic stability of Lotka-Volterra 3-species reaction-diffusion systems with time delays," Journal of Mathematical Analysis and Applications, vol. 281, no. 1, pp. 186-204, 2003. 


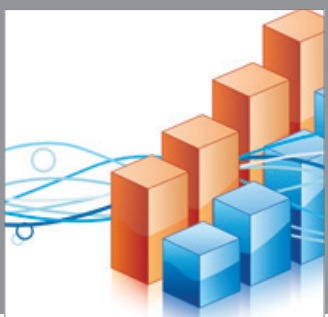

Advances in

Operations Research

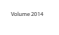

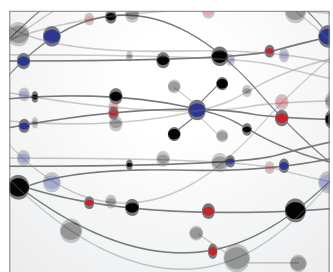

\section{The Scientific} World Journal
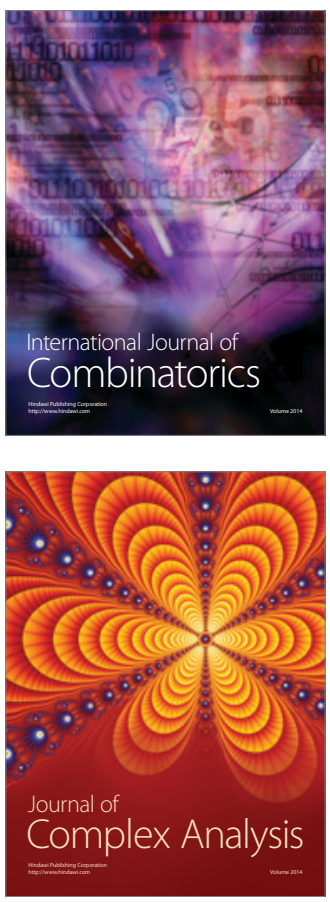

International Journal of

Mathematics and

Mathematical

Sciences
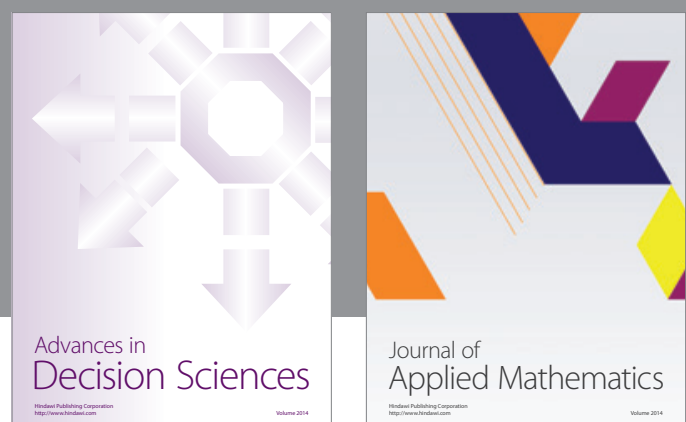

Journal of

Applied Mathematics
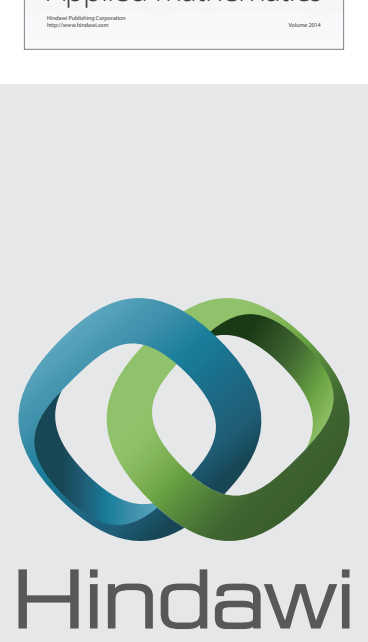

Submit your manuscripts at http://www.hindawi.com
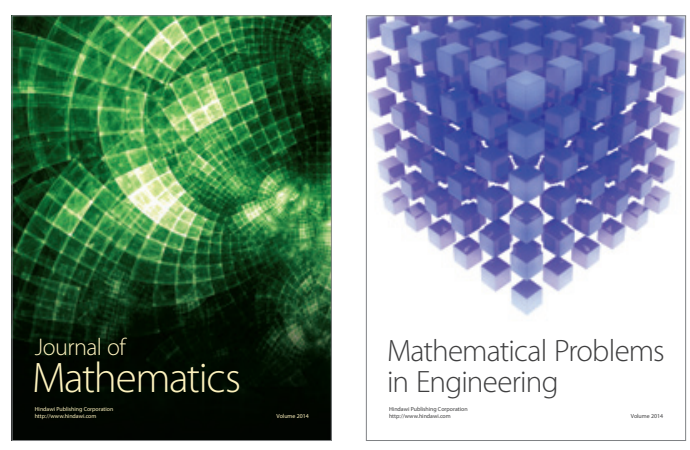

Mathematical Problems in Engineering
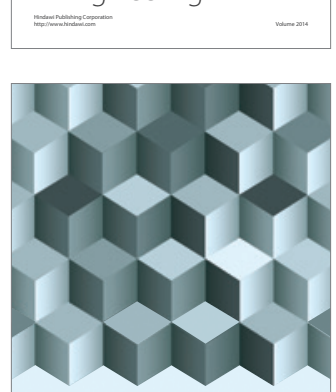

Journal of

Function Spaces
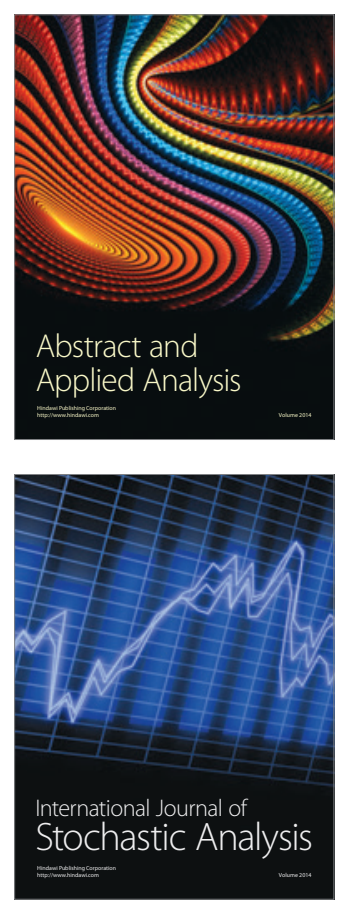

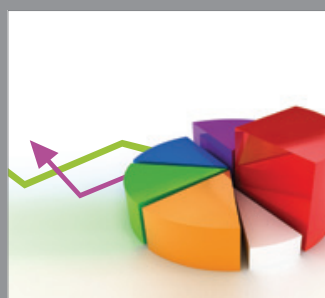

ournal of

Probability and Statistics

Promensencen
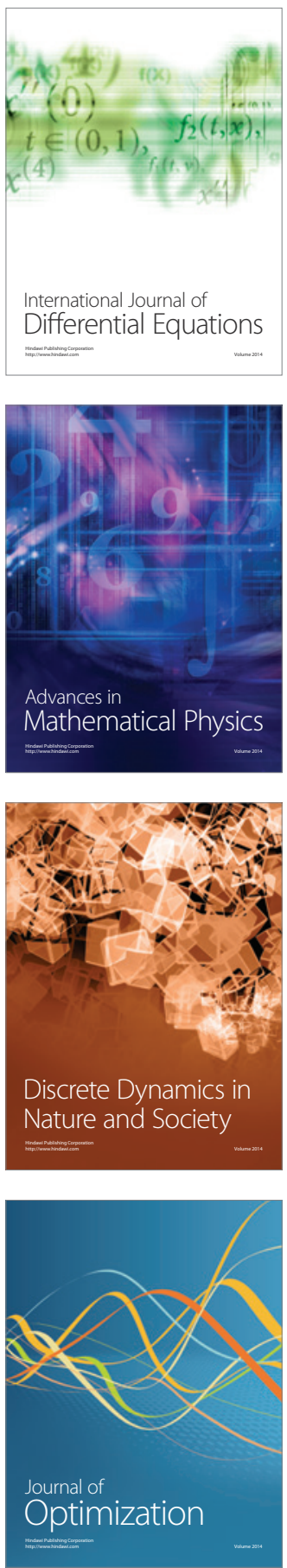